\title{
Corrigenda
}

\section{Kindling fluorescent proteins for precise in vivo photolabeling}

Dmitriy M. Chudakov, Vsevolod V. Belousov, Andrey G. Zaraisky, Vladimir V. Novoselov, Dmitry B. Staroverov, Dmitry B. Zorov, Sergey Lukyanov, and Konstantin A. Lukyanov

Nat. Biotechnol. 21, 191-194 (2003)

On page 191, information about a mutant was omitted. The last sentence of the third paragraph should read as follows:

Among the mutant proteins screened, the asCP A148G mutant with random substitutions F7L, Y26C, T30I, A142V (designated KFP1, GenBank accession no. AY233273) demonstrated the most remarkable properties, which make it suitable as a photoactivated fluorescent label.

On page 192, last full paragraph, the irradiation time for the irreversible KFP1 activation in mitochondria was listed incorrect. The sentence should read:

Brief irradiation (fast mode, 20 seconds) with $30 \%$ power green laser light induced irreversible kindling of KFP1 in mitochondria within the irradiated field.

\section{Immunofluorescent labeling of cancer marker Her2 and other cellular targets with semiconductor quantum dots}

Xingyong Wu, Hongjian Liu, Jianquan Liu, Kari N. Haley, Joseph A. Treadway, J. Peter Larson, Nianfeng Ge, Frank Peale, and Marcel P. Bruchez

Nat. Biotechnol. 21, 41-46 (2003)

In the Acknowledgments, a grant listing was omitted. The paragraph should state that the authors' work was also supported in part by grant 20000-00-4106A from the National Institute of Standards and Technology.

\section{Human zinc fingers as building blocks in the construction of artificial transcription factors}

Kwang-Hee Bae, Young Do Kwon, Hyun-Chul Shin, Moon-Sun Hwang, Eun-Hyun Ryu, Kyung-Soon Park, Hyo-Young Yang, Dong-ki Lee, Yangsoon Lee, Jinwoo Park, Heung Sun Kwon, Hyun-Won Kim, Byung-Il Yeh, Hyean-Woo Lee, Soon Hyung Sohn, Joonho Yoon, Wongi Seol, and Jin-Soo Kim

Nat. Biotechnol. 21, 275-280 (2003)

An author's name was misspelled. Soon Hyung Sohn should be Joon Hyung Sohn.

\section{Erratum}

\section{Decoding the literature on genetic variation}

Roger Coronini, Marie-Angèle de Looze, Pierre Puget, Gérard Bley, and Shyama V. Ramani

Nat. Biotechnol. 21, 21-29 (2003)

On page 21, the key to Figure 1 is incorrect. The colors denoting patents and scientific papers should be reversed: green should refer to patents and yellow to papers. 